\title{
Effects of Foraging Related Stimuli on OLM (Object Location Memory) and Perceptual Search in the Hunter-Gatherer Theory*
}

\author{
Espen A. Sjoberg, Geoff G. Cole, Luke T. Cannon \\ University of Essex, Essex, United Kingdom
}

\begin{abstract}
The hunter-gatherer theory suggests that a division of labor existed in early human settlements whereby men were predominantly hunters and women were predominantly gatherers. Support for this theory has come from the observation that females tend to perform better on tasks concerning object location memory, a skill required for successful gathering. We tested the hunter-gatherer theory through two experiments: (1) an OLM (object location memory) task where males and females were required to encode and recall the locations of animals, fruit, and neutral stimuli; and (2) a perceptual search task where males and females were required to search for either a fruit or an animal in an array of items. With OLM, we found the usual female advantage for neutral stimuli, but this difference was abolished with animal and fruit stimuli. The perceptual search task found no significant gender differences. These results therefore provide only partial support for the hunter gatherer theory.
\end{abstract}

Keywords: evolution, foraging stimuli, hunter-gatherer, sex differences, OLM (object location memory), perceptual search

\section{Introduction}

The hunter-gatherer theory (Silverman \& Eals, 1992) proposes an evolution of cognitive sex differences based on a division of labour in early human settlements. The basic tenet of the theory is that men hunted for game and women gathered edible plants and fruit, causing selection pressures to arise for the development of skills associated with each set of tasks. For instance, men would have developed skills required for navigating terrain and stalking prey. In contrast, women were more restricted to their immediate environment, and their gathering role would have caused a development of location memory in order to recall food locations more effectively for subsequent harvesting. Silverman and Eals (1992) developed a measure of OLM (object location memory), which required the location of items in arrays to be recalled. In accordance with their theory they found a female advantage in this task.

Much subsequent research has confirmed the female advantage in OLM. For instance, Silverman, Choi, and Peters (2007) observed the effect with approximately 250,000 participants from over 40 countries. Furthermore, in a meta-analysis of the female advantage the effect size was found to be $d=0.27$ (Voyer, Postma, Brake, \& Imperato-McGinley, 2007). However, this female advantage typically relies on specific experimental conditions. For instance, delaying the test phase by approximately a week will remove any sex differences observed (Honda \& Nihei, 2009). Manipulating distance also modulates the effect such that a male

\footnotetext{
*Acknowledgements: We thank Roger Grace for technical support and programming; Rachael Wilner for participant support, evaluation, and input; Phil Burke for input and discussions about hunter-gatherer theory. We also thank Rick O'Gorman, Leif Kennair, Max Krasnow, Russell Jackson, and Leda Cosmides for input.

Espen A. Sjoberg, MSc., Department of Psychology, University of Essex.

Geoff G. Cole, Ph.D., Department of Psychology, University of Essex.

Luke T. Cannon, BSc., Department of Psychology, University of Essex.
} 
advantage is observed in far visual space and a female advantage in near visual space (Saucier, Lisoway, Green, \& Elias, 2007). It has also been suggested that reducing the complexity of memory arrays may abolish the sex difference (Postma, Izendoorn, \& De Haan, 1998; Postma, Winkel, Tuiten, \& van Honk, 1999). Finally, the female advantage is more profound if implicit instructions are used. If participants are explicitly told to remember the location of items, the female advantage is typically reduced (Silverman \& Eals, 1992; Eals \& Silverman, 1994), though it is not entirely clear how reliable this pattern is or even why it occurs (Voyer et al., 2007).

The observed female advantage in OLM may rely on superior verbal memory. For instance, using items that are relatively hard to verbally encode will reduce the effect (Eals \& Silverman, 1994), and complex pictorial art or abstract figures will remove it completely (Chipman \& Kimura, 1998; Rahman, Bakare, \& Serinsu, 2011). Furthermore, increasing the ecological validity of the OLM paradigm also tends to reduce the female advantage (Cherney \& Ryalls, 1999; Iachini, Sergi, Ruggerio, \& Gnisci, 2005). One noteworthy exception is the study of New, Krasnow, Truxaw, and Gaulin (2007), which involved a real-world scenario of remembering food locations at a farmer's market. When participants were prompted to point in the direction of various food stalls from a viewpoint, they found that women could more accurately point in the direction of previously encountered food locations in the market compared to men.

Postma et al. (1998) argued that OLM is based on a representation that codes for absolute positioning, relative positioning, or a combination of the two. This is supported by the observation that moving items to previously empty locations rather than exchanging them around on the array will abolish the female advantage (James \& Kimura, 1997). Absolute positioning involves precision, and this will remove any female advantage and can even create a male advantage (Postma, Jager, Kessels, Koppeschaar, \& van Honk, 2004; Postma et al., 1999). With relative positioning, the female advantage in OLM persists as females appear to be better at assessing the position of items in relation to other items as opposed to their precise location. When items are moved to empty locations, as in James and Kimura (1997), OLM relies on a precision component and females no longer have an advantage.

Sex differences in OLM are also reflected in navigation skills. Choi and Silverman (2003) found that OLM performance predicted a preference for using landmarks when navigating long distances, but this effect was only significant with females. This suggests that when women navigate they depend largely on landmarks and their relative position. By contrast, men tend to employ Euclidean strategies; navigation based on geometric properties, distance, and three-dimensional directions. Women compensate for this by relying on verbally easier-to-label landmarks. Men will tend to use cardinal directions such as north and south to navigate, while women use relative directions such as left and right (Dabbs, Chang, Strong, \& Milun, 1998). The evolutionary cause for this is that men were hunters and navigated long ranges with fewer directional cues, while women stayed closer to camp and used landmarks to aid navigation.

\section{Object Location Memory and Foraging Stimuli}

Given the amount of work undertaken that has examined OLM in the context of the hunter-gatherer theory, it is somewhat surprising that little research has examined the influence of stimuli type on OLM. If spatial and memory sex differences have evolved due to labor division, one can posit that women would have developed a sensitivity to aspects of fruit stimuli and men to animal stimuli. Hulbert and Ling (2007) reported that women prefer colors signaling ripeness, especially reddish hues. McGlasson, Lorince, Crandall, and Todd (2013) analysed over 100 million photographs on the website Flickr and found that women showed a preference for the colours red and green in their own photographs. In addition, Neave, Hamilton, Hutton, Tildesley, and 
Pickering (2005) found that women are better than men at remembering the location of plants in a naturalistic environment. In the hunter-gatherer Hadza tribe, a population native to Tanzania, Berbesque, and Marlow (2009) found that the men prefer meat to berries, while the women show the opposite pattern.

However, only two previous studies have manipulated the stimuli in an OLM task such that they were evolutionary relevant. Cashdan, Marlowe, Crittenden, Porter, and Wood (2012) used a card-variant of the OLM paradigm to test the Hadza tribe. They used cards with pictures of local plant and animal life, but found no sex difference in performance depending on stimuli type. Krasnow et al. (2011) also used an OLM task to measure the effect of different stimuli types on performance. They found a female advantage in a stimuli category of "fruit on trees", but no such difference with animal stimuli. However, the authors did not observe the basic female advantage in conditions that presented non-evolutionarily relevant stimuli because they employed a version of OLM using absolute positioning, something which typically removes an overall female advantage (Postma et al., 2004). One can argue that the basic female advantage effect on relative OLM needs to be shown before any conclusions can be made with respect to evolutionary relevant stimuli and the hunter-gatherer theory.

\section{Perceptual Search and Foraging Stimuli}

In addition to the encoding and recall of object location, one can also argue that the hunter-gatherer theory predicts sex differences in the ability to find evolutionary relevant stimuli in a display. Specifically, the theory suggests that men and women would both employ a search strategy when foraging for game or fruit, respectively, but for different reasons: men for scanning terrain for potential game, while women would search plants for edible food. For this reason, hunter-gatherer theory predicts that men should be better than women at locating an animal in an array, while women should be better at locating fruit. Little research has been done regarding foraging-related stimuli on perceptual search, particularly in the context of sex differences. One study by Tipples, Young, Quinlan, Brooks, and Ellis (2002) examined reaction times to find fruit and animal stimuli, but did not investigate sex differences. They found that reaction times did not differ between animal and fruit stimuli. Another study investigated reaction time differences between animals and plants and found that people responded faster to animals than to plants (Lipp, Derakshan, Waters, \& Logies, 2004). Again, sex differences were not measured. The authors hypothesized that reaction times associated with animals should be shorter because animals are potentially dangerous predators with unpredictable trajectories. However, as sex differences were not investigated we cannot be sure if the advantage with animals was due to males scoring higher than females.

Stoet (2011) investigated sex differences in a perceptual search task and found that men were able to detect targets in an array of distracters faster than women. Differences in performance between animals and fruit were not investigated. However, in one search task fruit was as added as a potential target. No sex differences were observed in search time between fruit and neutral stimuli (such as letters). These results may suggest an overall male advantage at detecting targets, but with fruit stimuli this difference is removed.

\section{The Present Study}

The aim of the present study was to examine whether OLM and visual search is influenced by evolutionary relevant stimuli. As noted, the notion that humans have evolved as hunters and gatherers suggests a sexual asymmetry in cognitive abilities such that males will be particularly effective at processing animal stimuli and women will be particularly effective at processing fruit stimuli. In the first experiment, we employed the basic OLM paradigm in which male and female participants were required to encode and recall the locations of several object images. Importantly, there were three different classes of stimuli: animals, fruit, 
and neutral (or non-foraging stimuli). The neutral condition refers to any object that is not related to hunting or gathering (for example pencils, clocks, or coffee mugs). The vast majority of previous work on OLM has used a variety of objects unrelated to hunting or gathering. In accordance with previous research, women should outperform men on OLM tasks when the stimuli have not been manipulated to reflect hunting or gathering, i.e., the stimuli are neutral (Chipman \& Kimura, 1998; Voyer et al., 2007). However, as we aimed to use explicit instructions it is possible that the female advantage will be reduced and escape significance (Voyer et al., 2007). With respect to the animal and fruit conditions, the prediction is that a male improvement should occur for the animal stimuli compared to neutral stimuli, and a female improvement for the fruit stimuli because of their respective roles as game hunters are fruit gatherers in early human settlements.

In the second experiment, we assessed whether sex differences occur in perceptual search. Males and females were required to search for an animal or a fruit in an array of neutral objects. The hunter-gatherer theory posits the prediction that a male advantage may occur when searching for animal stimuli, and a female advantage when searching for fruit stimuli. In their respective hunter-gatherer roles, both men and women would have been required to use a searching process when looking for targets: Male hunters would have searched for prey among terrain, and female gatherers would have searched for fruit and vegetables among foliage. It is therefore possible that both genders would perform equally well at this task, but that an interaction should be found where men's performance increases with animal targets and women's performance increases with fruit targets.

\section{Experiment 1}

\section{Materials and Methods}

The experiment was approved by the University of Essex Psychology ethics committee.

A trial in the experiment consisted of six photographs randomly positioned within a uniform white background on the screen in a hexagonal design, similar to the approach by Krasnow et al. (2011). The stimuli were not mixed, but rather presented in categories, with each trial having six photographs from only one stimuli category. An example array can be seen in Figure 1. The size of each photo was $5 \times 3.5 \mathrm{~cm}$ in height and width. These were stock photos obtained via Google Images. With neutral objects, care was taken not to select stimuli that were gender-specific, such as masculine or feminine objects. As a result, neutral objects consisted of items such as pens or clocks. With animal photos there were no threatening predators such as snakes or lions to avoid evoking any fear responses. All animal photos were of potential prey such as deer, elk, or ducks, and all photos showed the animals clearly.

\section{Participants and Procedure}

The experiment was conducted on 76 adult participants (34 males, 42 females). Participants were instructed to memorize the location of the photos and then subsequently place them into the correct positions. There were no practice trials, but participants were shown two demonstration trials by the experimenter. Before the first trial instructions were also shown on the computer screen. Participants were told that there was no time limit and that they could change their mind before continuing to the next trial. Each trial began by displaying the six-item array of photos for five seconds (i.e., the encoding phase). Participants were then required to place the photos back into their respective positions (test phase). In the encoding phase there were small black frames around the photos, and in the testing phase these frames remained as reference points. A correct response consisted of positioning a photo where it had previously been displayed. There was a three second delay between the test phase and a new trial.

The experiment employed a $2 \times 3$ mixed design with sex as the between-subjects variable and stimuli type as the within-subjects variable. For the stimuli type variable, there were three levels: neutral, animal, and fruit. 
The measurement consisted of the number of photos correctly positioned in the testing phase. There were a total of 15 trials, five trials for each of the three conditions. The presentation order of the trials was randomized.

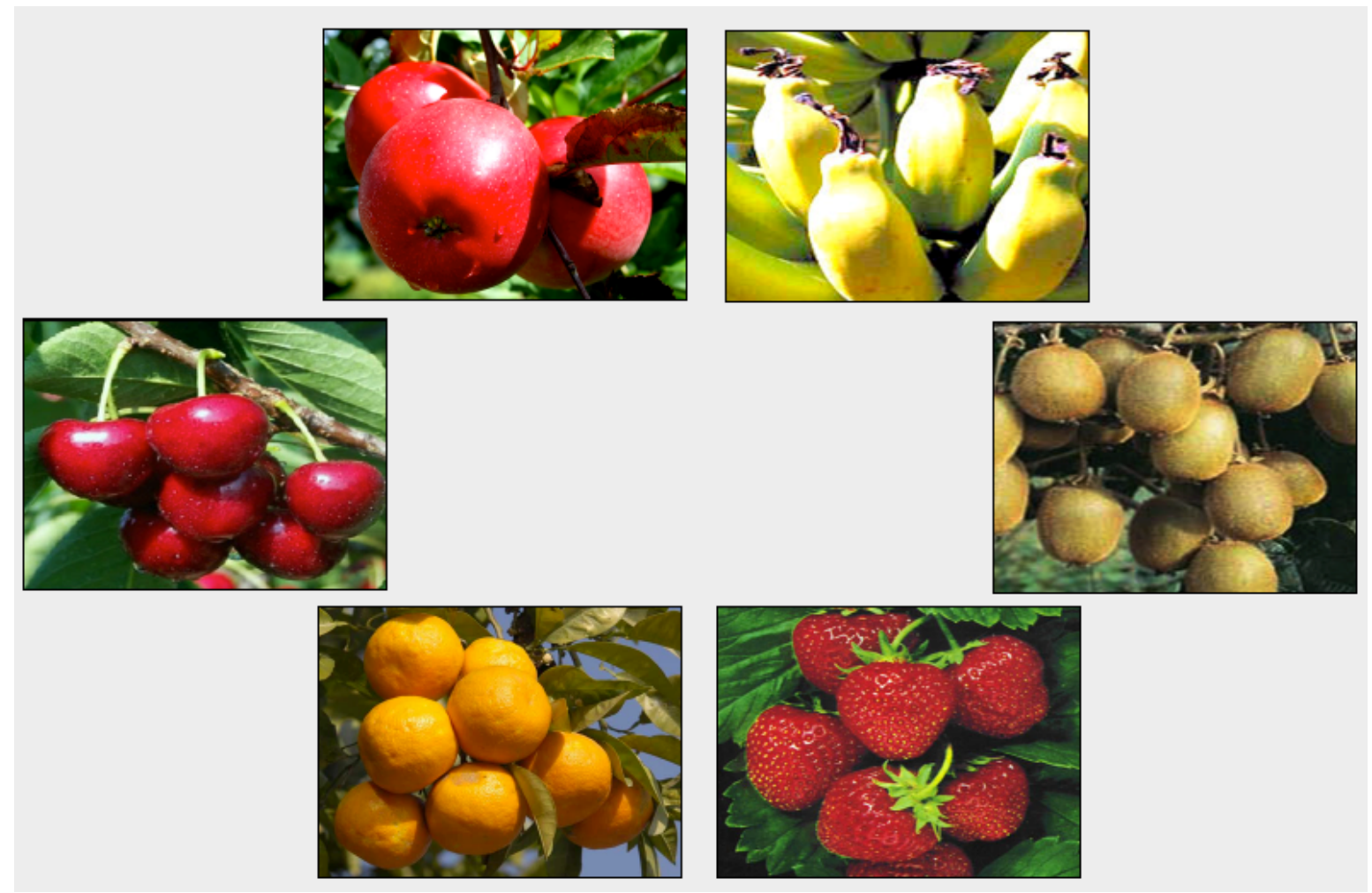

(a) Stimulus array presentation.
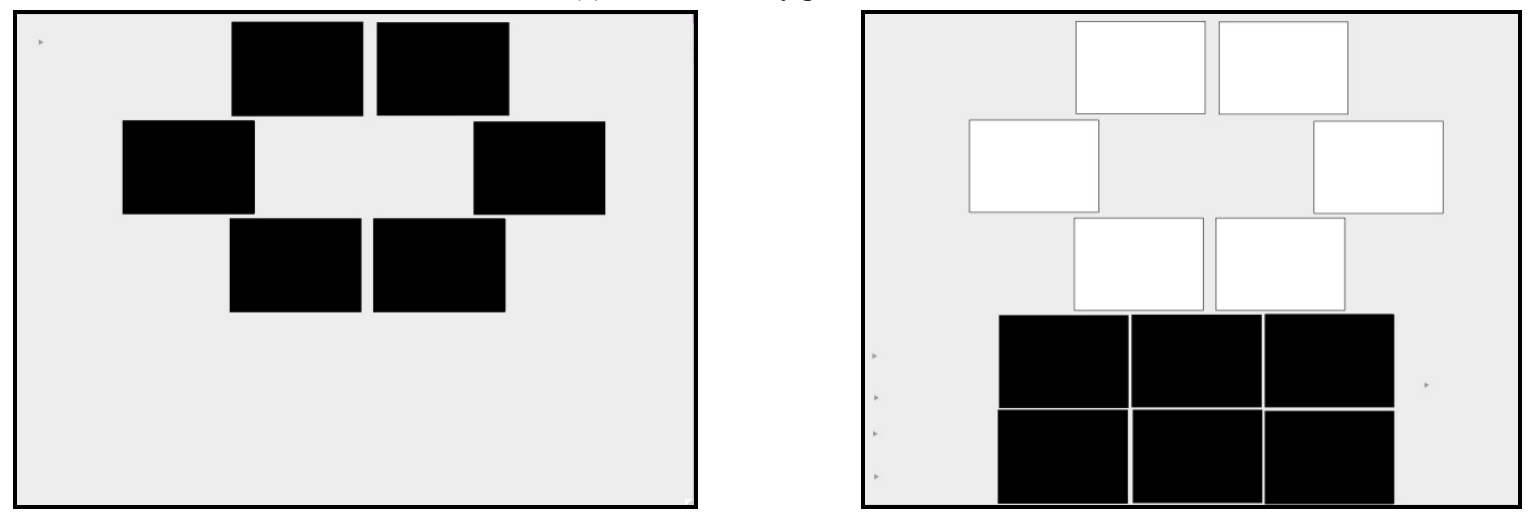

(b) Location memory task.

Figure 1. Participants were presented with an array (a) of objects that consisted of either animals, fruit, or objects in a neutral category (that are not relevant to hunting or gathering). Participants were then required to return the objects to their initial respective places (b).

\section{Results}

The mean number of photos correctly positioned is summarized in Figure 2. A $2 \times 3$ mixed ANOVA, with sex as the between-participants variable and stimuli type as the within-participants variable, found no main effect of sex, $F_{(1,74)}=0.153, p=0.697$, and no main effect of stimuli type, $F_{(2,148)}=0.732, p=0.483$. A significant interaction was however found between sex and stimuli type, $F_{(1,148)}=3.081, p=0.049, \eta_{p}{ }^{2}=0.04$.

A follow-up independent samples $t$-test revealed that, as predicted, females showed greater OLM performance for neutral stimuli, $t_{(74)}=1.724, p<0.05, d=0.4$. There were however no significant sex differences for either of the other two stimuli types, both $t_{(74)}<0.47, p \mathrm{~s}>0.64$. 
In sum, Experiment 1 shows the usual female OLM advantage with neutral stimuli, with an effect size in line with the previous literature (Voyer et al., 2007). However, this advantage was removed with animal and fruit stimuli.

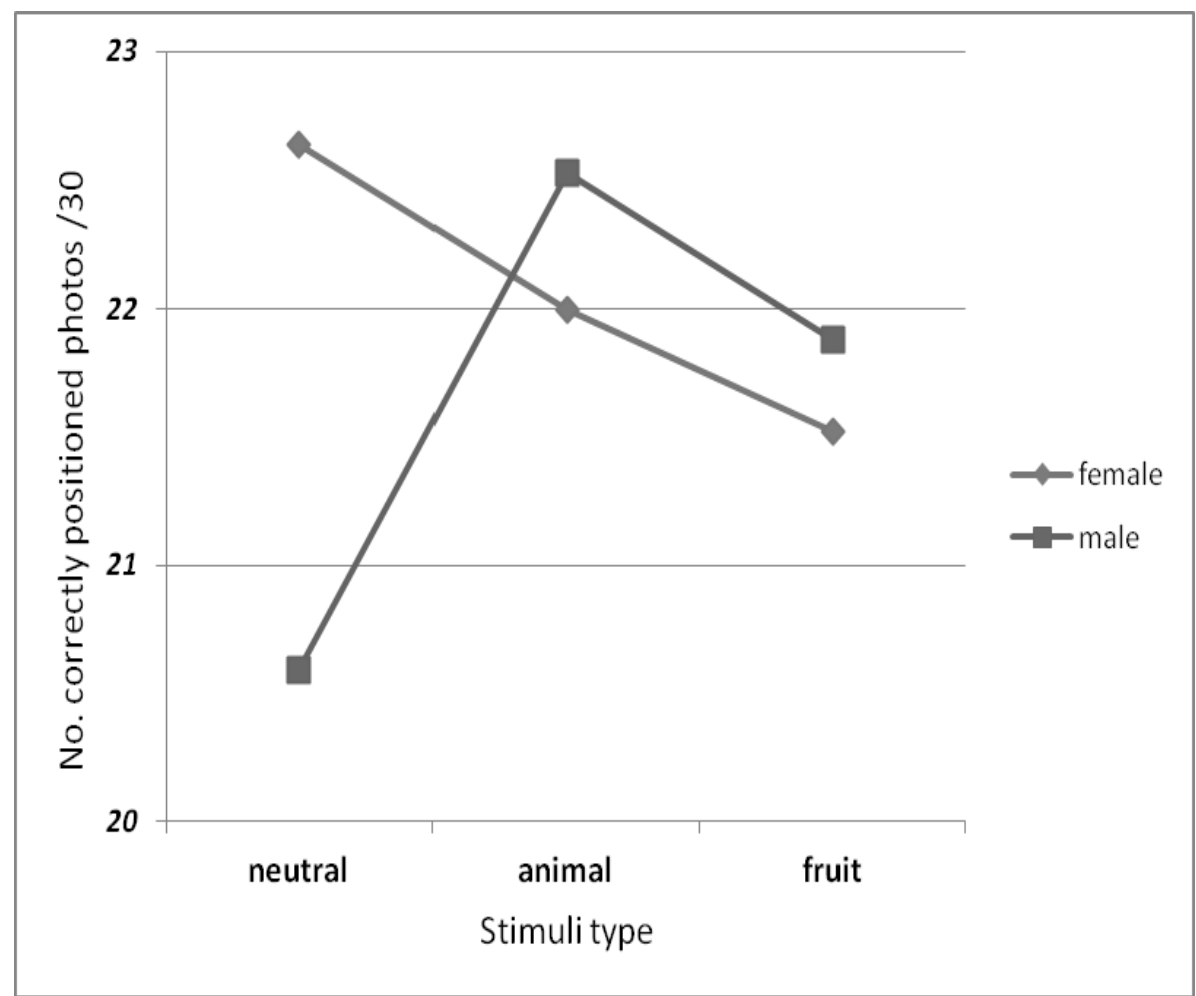

Figure 2. Means of number of correctly positioned photos in males and females across stimuli type. For males, the mean (95\% confidence interval) for number of correctly positioned photos was 20.6 (18.8-22.35) for neutral, 22.5 (20.8-24.2) for animals, and 21.9 (20-23.8) for fruit. For females, it was 22.6 (21.1-24.2) for neutral, 22.0 (20.5-23.5) for animals, and 21.5 (19.8-23.2) for fruit.

\section{Experiment 2}

\section{Materials and Methods}

A trial consisted of 20 photographs positioned in a $5 \times 4$ format. The size of each photo on the screen was $4 \times 3.5 \mathrm{~cm}$ (length $\times$ height). The array was centered on the screen, and there were a total of 40 arrays. Half the arrays had 20 pictures of neutral objects (no targets), and the other half had 19 pictures of neutral objects with one photo of a target, which was a fruit or an animal. We can see Figure 3 for an example array. Targets were presented at random locations within the array. For fruit and animal stimuli, photos from Experiment 1 were used. For neutral stimuli, randomly selected objects from a variety of categories were chosen, which were taken from Google Images, though care was taken not to include any photos of humans, animals, or of plants that could potentially carry fruit.

The order of the trials was randomized. Of main interest was the measurement of reaction time to correctly detect targets. However, the number of targets correctly detected was also recorded, and if a participant "detected" a target in a neutral array (i.e., target absent) this was recorded as a false positive.

\section{Participants and Procedure}

The experiment was conducted on 53 adult participants ( 24 males, 29 females).

They were instructed to watch the screen and detect either an animal or a fruit as fast and as accurate as possible clicking one of two possible buttons, labeled "Yes" or "No". There were no practice trials, but 
participants were shown a brief demonstration by the experimenter and instructions were also presented on the screen. Participants were told that all pictures of fruit and animals would show the target clearly and there should be no ambiguous photos (such as distant bushes that potentially carried berries). The experiment was presented in counter-balanced blocks with each block having either the animal or fruit as targets. There was a three second delay between response and a new trial. Upon completing a block, instructions were given again on the computer screen and the participants were allowed to take a break if they wanted.
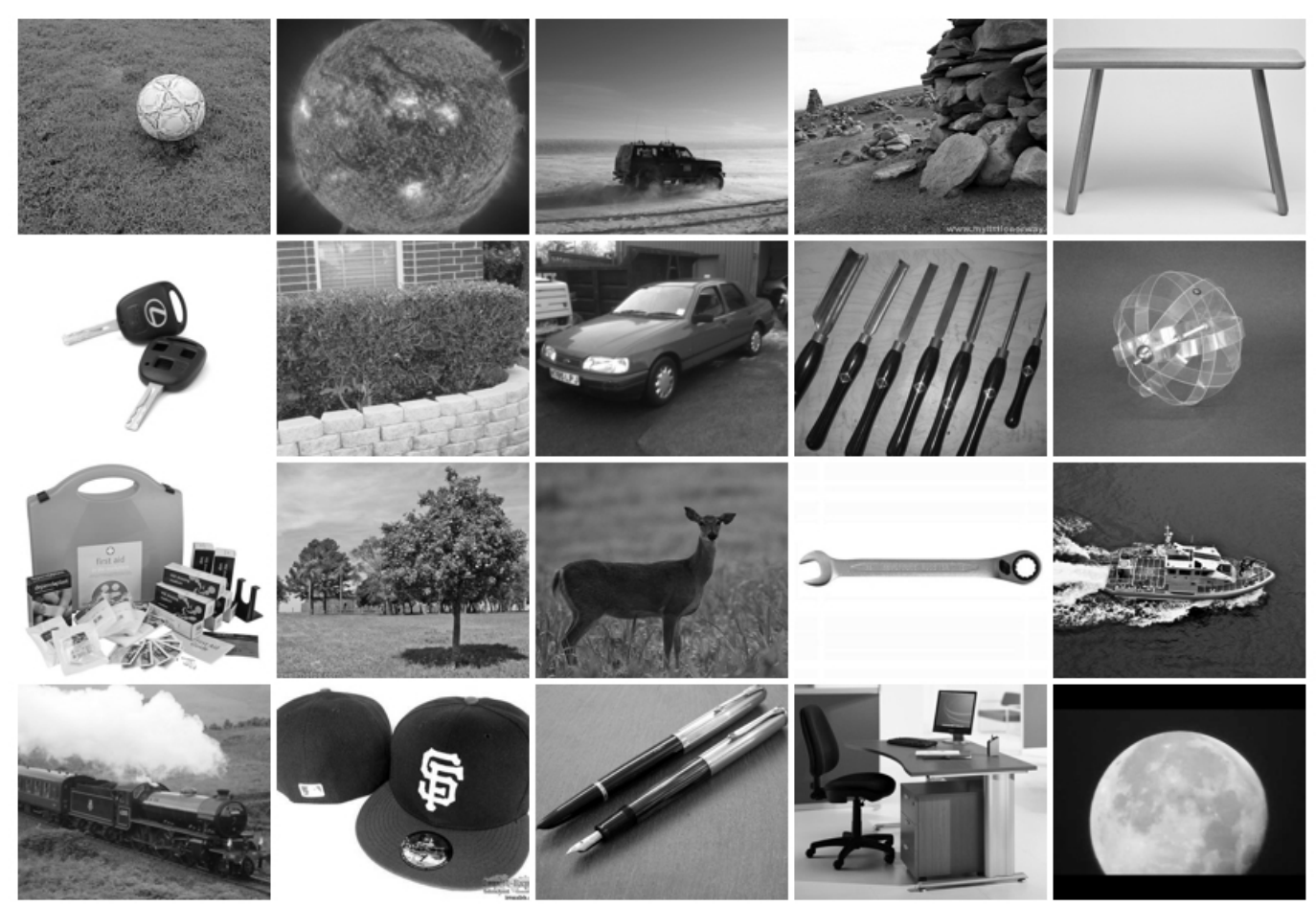

Figure 3. Example array from Experiment 2, consisting of 19 neutral objects and one animal. Participants are told to respond "Yes" as soon as they detected the animal, or "No" if they were reasonably sure no animal was present.

\section{Results}

Reaction time. The mean reaction time was calculated separately for each participant. Reaction time was measured in milliseconds, and the mean reaction time for stimuli type by sex is summarized in Table 1.

Table 1

Mean RT (Reaction Time) in Milliseconds for Fruit and Animal Targets. Mean Number of Correctly Identified Targets. Mean Number of FP (False Positives) Found, Displayed in Percentage of Responses

\begin{tabular}{lllc}
\hline & Male & Female & Cohen's $d$ \\
\hline Fruit (RT) & $1,153(407)$ & $1,122(319)$ & 0.09 \\
Animal (RT) & $1,240(407)$ & $1,098(229)$ & 0.45 \\
Fruit (No. correct) & $8.9(1.3)$ & $9.2(1.0)$ & -0.27 \\
Animal (No. correct) & $8.9(1.4)$ & $9.1(0.9)$ & -0.18 \\
Fruit FP & $2.3 \%(0.43)^{*}$ & $0 \%(0)^{*}$ & $0.80^{*}$ \\
Animal FP & $0.9 \%(0.29)$ & $2.1 \%(0.42)$ & -0.33 \\
\hline
\end{tabular}

Note." $p=0.01$.

A $2 \times 2$ mixed analysis of variance with sex as the between-subject variable and stimuli type as the within-subjects variable was conducted. No main effect of sex was found, $F_{(1,51)}=1.017, p=0.318, \eta_{p}{ }^{2}=$ 0.020 , and no main effect of stimuli type, $F_{(1,51)}=0.641, p=0.427, \eta_{p}{ }^{2}=0.012$. There was also no significant 
interaction, $F_{(1,51)}=1.936, p=0.17, \eta_{p}{ }^{2}=0.037$, thus men and women correctly detected targets equally fast regardless of stimuli type.

Target detection accuracy. A $2 \times 2$ mixed analysis of variance with sex as the between-subject variable and stimuli type as the within-subjects variable was conducted. No main effects were found for sex, $F_{(1,51)}=$ $0.878, p=0.353, \eta_{p}{ }^{2}=0.017$, nor stimuli type, $F_{(1,51)}=0.74, p=0.787, \eta_{p}{ }^{2}=0.001$. There was also no significant interaction, $F_{(1,51)}=0.74, p=0.787, \eta_{p}{ }^{2}=0.001$. Men and women correctly detected an equal amount of targets.

False positives. With respect to false positives, data from three participants were discarded as their responses were more than three standard deviations above the mean. A $2 \times 2$ mixed analysis of variance with sex as the between-subject variable and stimuli type as the within-subjects variable was conducted. No main effect was found for sex, $F_{(1,48)}=0.473, p=0.495, \eta_{p}{ }^{2}=0.10$, and no main effect of stimuli type was found either, $F_{(1,48)}=0.492, p=0.487, \eta_{p}{ }^{2}=0.10$. However, an interaction between sex and stimuli type was found, $F_{(1,48)}=9.953, p=0.003, \eta_{p}{ }^{2}=0.172$. Despite significance, false positives were relatively rare in the sample with $78 \%$ of participants making no such errors, and participants who did make false positives only made one (excluding the three discarded participants).

An exploratory follow-up independent samples $t$-test revealed that women showed significantly less false positives with fruit than men, $t_{(48)}=2.812, p=0.007, d=0.801$. However, only five males made a false positive error, compared to no females, so despite significance the impact on reaction time was minimal.

In sum, men and women did not differ in their search speed for animals and fruit, nor did they differ in amount of targets correctly detected. Men made more false positives with fruit than women did, though such errors were rare.

\section{General Discussion}

Our experiments show that in both OLM and perceptual search there was no difference in memory performance or reaction time between men and women with either fruit or animal stimuli. This does not support the hunter-gatherer theory, which should predict a male advantage with animal stimuli and a female advantage with fruit stimuli. However, the hunter-gatherer theory is only partially supported by the finding that males perform better in OLM when presented with animal stimuli compared to neutral stimuli, but this increased performance remained with fruit stimuli.

In the OLM experiment the female advantage in the neutral condition concurs with previous research in which non-evolutionary relevant stimuli are typically used. In a meta-analysis by Voyer et al. (2007) an effect size of $d=0.27$ was found in favor females on OLM. In our experiment we found a female advantage with the effect size $d=0.40$, in line with the literature. However, it should be noted that our experiment uses explicit instructions, and the female advantage is usually reduced using this encoding context (Voyer et al., 2007). Our choice of encoding context may explain the absence of a significant main effect of sex in the OLM task, but it does not explain why women outperformed men with neutral objects that were unrelated to hunting or gathering, but not with foraging-related objects. That women and men performed equally well with animals and fruit is consistent, however, with the findings of Cashdan et al. (2012), who also found no sex difference in performance between the stimuli using explicit instructions.

In Experiment 2, we found that men and women did not differ significantly in their search speed for fruit and animals. This finding is similar to that found by Tipples, Young, Quinlan, Broks, and Ellis (2002) who reported no difference in reaction time between animal and fruit stimuli. In addition, Stoet (2011) did not find any difference in search speed between fruit and neutral stimuli. However, Stoet also observed a significant 
male advantage in his search task, while in our study the pattern suggested an overall female advantage, though this was not significant. It may be that fruit and animal stimuli carry equal evolutionary value, but for potentially different reasons. From an evolutionary point of view, fruit may have a purely survivalist value as a food source, while animals are associated with potential predators and movement detection as well as a source of food. As these qualities are equally important for both genders, this may be why no sex difference is observed between them.

The increase in male OLM performance in the animal stimuli condition, resulting in no difference with females, could be due to the animate monitoring hypothesis put forward by New, Cosmides, and Tooby (2007; see also the "behavioral urgency hypothesis", Cole \& Kuhn, 2009, 2010; Franconeri \& Simons, 2003). This suggests that naturally animate organisms such as animals and humans are subject to increased processing due to a survival advantage. Predator animals, for example, may display sudden and dangerous trajectory changes, and so it is beneficial to react faster to these stimuli. Indeed, humans show faster reaction times to animals than to inanimate objects such as plants (Lipp, Derakshan, Waters, \& Logies, 2004), and even mobile manmade objects such as cars (New et al., 2007). Of course, fruit stimuli are immobile and this hypothesis would suggest that animal stimuli should create a higher performance compared to fruit. However, this is not found in either the OLM task or the perceptual search task. Therefore, the underlying mechanism for animals could have caused an increase in performance with animal stimuli, but a different mechanism caused the same effect with fruit. This brings forth the notion that the evolutionary causes for the reaction mechanisms associated with fruit and animal stimuli are not mutually exclusive.

The reason why fruit would also enhance performance in males requires further examination, but it is possible that fruit carries evolutionary importance because it is a food source. If the increased performance seen with fruit was a result of labor divisions then men would not be expected to perform higher when dealing with fruit stimulus. Instead, it is likely that any underlying mechanism for fruit stimuli stems from a much deeper evolutionary origin than suggested by hunter-gatherer theory. As fruit has been an important food source for many animals across the evolutionary history, it is likely to carry equal importance in both men and women. That men and women were equally fast at detecting both fruit and animals support this notion. Even if women were gatherers in more recent human history, prior to this no labor division existed and a fruit mechanism could be the result of opportunistic searching for fruit between both sexes, much further back in human evolutionary history.

This relates to criticisms suggesting that the typical female advantage observed in OLM may not be due to a labor division in early human settlements. For instance, rats also display a female advantage in OLM, yet rats do not have a division of labor (Saucier, Shultz, Keller, Cook, \& Binsted, 2008). This suggests that the observed sex differences in OLM and mental rotation in previous studies are likely to stem from a much longer evolutionary history. Stoet (2011) also argues against the hunter-gatherer theory, proposing that the division of labor in early human settlements may not have been due to skill, but convenience. Men were not necessarily hunters because they were better at it, but, for example, because child rearing prohibited women from hunting. Kuhn and Stiner (2006) emphasize that roles in a hunter-gatherer society were learned. There were also female hunters, especially in families with widows or without sons.

Jones, Braithwaite, and Healy (2003) reviewed several evolutionary theories that attempted to explain sex differences in spatial abilities. They argued that the often inconsistent evidence for hunter-gatherer theory does not fit the assumption of a gender-specific labor division and as such they propose that the Silverman and Eal's (1992) theory is no longer viable. Jones et al. (2003) concluded that the most fitting theory to explain sex differences in spatial abilities was the range size theory, which involved males dispersing over large territories 
in order to optimize mating and father more offspring. This is similar to the Ecuyer-Dab and Roberts' (2004) theory, which suggested that male competition, mating, and extensive ranging would explain male advantages in spatial abilities. In contrast, females would focus on safeguarding their survival with restricted navigation and concentration of nearby spatial cues, which could account for a why women perform better in OLM and in near space.

In sum, the present work has provided only partial support for the hunter-gatherer theory of sex differences in OLM. The usual female advantage observed in OLM tasks was abolished when participants were required to remember the locations of animal and fruit stimuli. A perceptual search task found no difference between men and women when searching for animals or fruit in an array.

\section{References}

Berbesque, J. C., \& Marlowe, F. W. (2009). Sex differences in food preferences of Hadza hunter-gatherers. Evolutionary Psychology, 7(4), 601-616.

Cashdan, E., Marlowe, F. W., Crittenden, A., Porter, C., \& Wood, B. M. (2012). Sex differences in spatial cognition among Hadza foragers. Evolution and Human Behaviour, 33(4), 274-284.

Cherney, I. D., \& Ryalls, B. O. (1999). Gender-linked differences in the incidental memory of children and adults. Journal of Experimental Child Psychology, 72, 305-328.

Chipman, K., \& Kimura, D. (1998). An investigation of sex differences on incidental memory for verbal and pictorial material. Learning and Individual Differences, 10(4), 259-272.

Choi, J., \& Silverman, I. (2003). Processes underlying sex differences in route-learning strategies in children and adolescents. Personality and Individual Differences, 34, 1153-1166.

Cole, G. G., \& Kuhn, G. (2010). Attentional capture by object appearance and disappearance. Quarterly Journal of Experimental Psychology, 63, 147-159.

Cole, G. G., \& Kuhn, G. (2009). Appearance matters: Attentional orienting by new objects in the precuing paradigm. Visual Cognition, 17, 755-776.

Dabbs, J. M., Chang, E. L., Strong, R. A., \& Milun, R. (1998). Spatial ability, navigation strategy, and geographical knowledge among men and women. Evolution and Human Behaviour, 19(2), 89-98.

Eals, M., \& Silverman, I. (1994). The hunter-gatherer theory of spatial sex differences: Proximate factors mediating the female advantage in recall of object arrays. Ethology and Sociobiology, 15, 95-105.

Ecuyer-Dab, I., \& Roberts, M. (2004). Have sex differences in spatial ability evolved from male competition for mating and female concern for survival? Cognition, 91, 221-257.

Franconeri, S. L., \& Simons, D. J. (2003). Moving and looming stimuli capture attention. Perception \& Psychophysics, 65, 999-1010.

Honda, A., \& Nihei, Y. (2009). Sex differences in object location memory: The female advantage if immediate detection of changes. Learning and Individual Differences, 19, 234-237.

Hulbert, A. C., \& Ling, Y. (2007). Biological components of sex differences in color preference. Current Biology, 17(16), 623-625.

Iachini, T., Sergi, I., Ruggiero, G., \& Gnisci, A. (2005). Gender differences in object location memory in a real three-dimensional environment. Brain and Cognition, 59, 52-59.

James, T. W., \& Kimura, D. (1997). Sex differences in remembering the locations of objects in an array: Location-shift versus location-exchanges. Evolution and Human Behavior, 18, 155-163.

Jones, C. M., Braithwaite, V. A., \& Healy, S. D. (2003). The evolution of sex differences in spatial ability. Behavioral Neuroscience, 117(3), 403-411.

Krasnow, M. M., Truxaw, D., Gaulin, S. J. C., New, J., Ozono, H., Uono, S.,... Minemoto, K. (2011). Cognitive adaptations for gathering-related navigation in humans. Evolution and Human Behavior, 32, 1-12.

Kuhn, S. L., \& Stiner, M. C. (2006). What's a mother to do? The division of labor among neandertals and modern humans in Eurasia. Current Anthropology, 47(6), 953-981.

Lipp, O. V., Derakshan, N., Waters, A. M., \& Logies, S. (2004). Snakes and cats in the flower bed: Fast detection is not specific to pictures of fear-relevant animals. Emotion, 4(3), 233-250.

McGlasson, C., Lorince, J., Crandall, D., \& Todd, P. (2013). Exploring sex differences in color preference using online resources. Paper presented at the Human Behaviour and Evolution Conference, July 17-20, Miami, United States. 
Neave, N., Hamilton, C., Hutton, L., Tildesley, N., \& Pickering, A. T. (2005). Some evidence of a female advantage in object location memory using ecologically valid stimuli. Human Nature, 16(2), 146-163.

New, J., Cosmides, L., \& Tooby, J. (2007). Category-specific attention for animals reflect ancestral priorities, not expertise. Proceedings of the National Academy of Sciences, 104(42), 16598-16603.

New, J., Krasnow, M., Truxaw, D., \& Gaulin, S. J. C. (2007). Spatial adaptations for plant foraging: Women excel and calories count. Proceedings of the Royal Society B, 274, 2679-2684.

Postma, A., Izendoorn, R., \& De Haan, E. H. F. (1998). Sex differences in object location memory. Brain and Cognition, 26, 334-345.

Postma, A., Jager, G., Kessels, R. P. C., Koppeschaar, H. P. F., \& van Honk, J. (2004). Sex differences for selective forms of spatial memory. Brain and Cognition, 54, 24-34.

Postma, A., Winkel, J., Tuiten, A., \& van Honk. (1999). Sex differences and menstrual cycle effects in human spatial memory. Psychoneuroendocrinology, 24, 175-199.

Rahman, Q., Bakare, M., \& Serinsu, C. (2011). No sex differences in spatial location memory for abstract design. Brain and Cognition, 76, 15-19.

Saucier, D. M., Lisoway, A., Green, S., \& Elias, L. (2007). Female advantage for object location memory in peripersonal but not extrapersonal space. Journal of the International Neuropsychological Society, 13, 683-686.

Saucier, D. M., Shultz, S. R., Keller, A. J., Cook, C. M., \& Binsted, G. (2008). Sex differences in object location memory and spatial navigation in Long-Evan rats. Animal Cognition, 11, 129-137.

Silverman, I., Choi, J., \& Peters, M. (2007). The hunter-gatherer theory of sex differences in spatial abilities: Data from 40 countries. Archives of Sexual Behaviour, 36, 261-268.

Silverman, I., \& Eals, M. (1992). Sex differences in spatial abilities: Evolutionary theory and data. In J. H. Barkows, L. Cosmides, \& J. Tooby (Eds.), The adapted mind: Evolutionary psychology and the generation of culture (pp. 531-549). New York: Oxford Press.

Stoet, G. (2011). Sex differences in search and gathering skills. Evolution and Human Behaviour, 32, 416-422.

Tipples, J., Young, A. W., Quinlan, P., Broks, P., \& Ellis, A. W. (2002). Searching for threat. The Quarterly Journal of Experimental Psychology A: Human Experimental Psychology, 55(3), 1007-1026.

Voyer, D., Postma, A., Brake, B., \& Imperato-McGinley, J. (2007). Gender differences in object location memory: A meta-analysis. Psychonomic Bulletin and Review, 14(1), 23-38. 